I N S T I T U T O

$\mathrm{DE}$

M E D I C I N A

T R O P I C A L

$\mathrm{DE}$

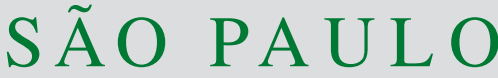

JOURNAL OF THE SÃO PAULO

INSTITUTE OF TROPICAL MEDICINE

1Universidade de São Paulo, Faculdade de Medicina de Ribeirão Preto, Programa de Mestrado em Oncologia Clínica, CélulasTronco e Terapia Celular, Ribeirão Preto, São Paulo, Brazil

${ }^{2}$ Universidade de São Paulo, Faculdade de Medicina de Ribeirão Preto, Hemocentro de Ribeirão Preto, Ribeirão Preto, São Paulo, Brazil

${ }^{3}$ Centre National de Référence Risques Infectieux Transfusionnels, Institut National de la Transfusion Département d'études des Agents Transmissibles par le Sang, Paris, France

${ }^{4}$ Institut Pasteur, Unité Environnement et Risques Infectieux, Cellule d'Intervention Biologique d'Urgence, Paris, France

${ }^{5}$ Universidade de São Paulo, Faculdade de Medicina de Ribeirão Preto, Departamento de Genética, Ribeirão Preto, São Paulo, Brazil

${ }^{6}$ Universidade de São Paulo, Faculdade de Medicina de Ribeirão Preto, Departamento de Clínica Médica, Ribeirão Preto, São Paulo, Brazil

Correspondence to: Svetoslav Nanev Slavov

Universidade de São Paulo, Faculdade de Medicina de Ribeirão Preto, Hemocentro de Ribeirão Preto, Rua Tenente Catão Roxo, 2501, CEP 14051-060, Ribeirão Preto, SP, Brazil

E-mail: svetoslav.slavov@ hemocentro.fmrp.usp.br

Received: 22 January 2021

Accepted: 18 April 2021

\section{Deep sequencing applied to the analysis of viromes in patients with beta-thalassemia}

\author{
Ian Nunes Valença ${ }^{1,2}$, Rafael dos Santos Bezerra ${ }^{1,2}$, Kamila Chagas Peronni², \\ Virginie Sauvage ${ }^{3}$, Mathias Vandenbogaert ${ }^{4}$, Valérie Caro ${ }^{4}$, Wilson Araújo \\ da Silva Junior ${ }^{5}$, Dimas Tadeu Covas ${ }^{2,6}$, Ana Cristina Silva-Pinto ${ }^{2}$, Syria \\ Laperche $^{3}$, Simone Kashima ${ }^{2}$, Svetoslav Nanev Slavov ${ }^{\circledR 2,6}$
}

\section{ABSTRACT}

To date, blood banks apply routine diagnosis to a specific spectrum of transfusiontransmitted viruses. Even though this measure is considered highly efficient to control their transmission, the threat imposed by emerging viruses is increasing globally, which can impact transfusion safety, especially in the light of the accelerated viral discovery by novel sequencing technologies. One of the most important groups of patients, who may indicate the presence of emerging viruses in the field of blood transfusion, is the group of individuals who receive multiple transfusions due to hereditary hemoglobinopathies. It is possible that they harbor unknown or unsuspected parenterally-transmitted viruses. In order to elucidate this, nucleic acids from 30 patients with beta-thalassemia were analyzed by Illumina nextgeneration sequencing and bioinformatics analysis. Three major viral families: Anelloviridae, Flaviviridae and Hepadnaviridae were identified. Among them, anelloviruses were the most representative, being detected with high number of reads in all tested samples. Human Pegivirus 1 (HPgV-1, or GBV-C), Hepatitis B Virus (HBV) and Hepatitis C Virus (HCV) were also identified. HBV and HCV detection was expected due to the high seroprevalence in patients with beta thalassemia. Our results do not confirm the presence of emerging or unsuspected viruses threatening the transfusion safety at present, but can be used to actively search for viruses that threaten blood transfusion safety. We believe that the application of viral metagenomics in multiple-transfused patients is highly useful to monitor possible viral transfusion threats and for the annotation of their virome composition.

KEYWORDS: Metagenomics. Next-generation sequencing. Beta-thalassemia. Virome. Hemotherapy. Emerging viruses.

\section{INTRODUCTION}

Blood Transfusion Services worldwide apply highly sensitive diagnostic tests to detect Human Immunodeficiency virus (HIV), Hepatitis B Virus (HBV), Hepatitis $\mathrm{C}$ virus (HCV), syphilis, and in some countries Human T-cell lymphotropic virus (HTLV) and Chagas disease as well. Therefore, hemotherapeutic procedures including blood transfusion are considered safe. Although the transfusion risk of transmission of routinely tested viral agents has been dramatically reduced, even the most sensitive detection techniques cannot guarantee a "zero risk". Therefore, occasional transfusion transmission of routinely tested agents is observed ${ }^{1}$ mainly due to circulation of novel mutation variants and/or the presence of very low viral load levels such as the ones reported in occult $\mathrm{HBV}$ infection ${ }^{2,3}, \mathrm{HCV}^{4}$ and $\mathrm{HIV}^{5}$. 
Apart from the risk of transfusion transmission of the routinely tested viruses, there is a risk of parenteral transmission of virtually any viral agent that will thereafter establish viremia. In this respect, emerging viruses which can threaten the safety of blood transfusion are especially important. The list of these viral agents is extensive ${ }^{6}$ and includes typical arboviral agents with short-termed viremia like dengue (DENV) ${ }^{7,8}$ and Zika viruses $(\mathrm{ZIKV})^{9}$ and others with prolonged viremic phases like Hepatitis E virus $(\mathrm{HEV})^{10,11}$ and Parvovirus type 4 (PARV4) ${ }^{12}$.

Multiple-transfused patients with hereditary hemoglobinopathies are at high risk of acquiring parenterally-transmitted viruses. Historically, these patients demonstrate higher prevalences of HIV, HCV and $\mathrm{HBV}^{13}$ compared to the general population. Moreover, due to frequent transfusions, one may infer that unsuspected viral infections which are not routinely tested in Blood Transfusion Services can also be present.

Considering that it is impossible to test all possible infections that can threaten transfusion safety, a suitable approach would be the virome examination of multiple -transfused patients through metagenomic High Throughput Sequencing (mHTS). This approach gives a detailed information regarding the overall viral abundance and diversity in any type of clinical sample, thus speeding up the discovery of emerging viruses ${ }^{14,15}$. Therefore, the use of mHTS for evaluating the virome in patients receiving multiple transfusions can provide important information on the presence of unsuspected viral infections that can threaten blood transfusion.

The aim of our study was to annotate the virome of patients with beta-thalassemia major in order to search for viral agents that can threat blood transfusion safety. The choice of this group was based on the following reasons: (i) Historically, patients with beta thalassemia present with higher indexes of transfusion-transmitted diseases, which have been reduced nowadays, but continue elevated compared to the general population. This demonstrates that patients with beta-thalassemia are under a constant threat of acquiring parenterally-transmitted infections, especially the emerging ones. (ii) Presence of endemic viral diseases in the examined region like dengue and Zika infections that are transmitted by arthropod vectors but might also be transmitted by blood transfusion.

\section{MATERIALS AND METHODS}

\section{Study population}

In the present study, we evaluated the virome of 30 patients with beta-thalassemia, who sought medical attention at the hemoglobinopathies outpatient clinic of the Blood Center of Ribeirao Preto, Faculty of Medicine of Ribeirao Preto, University of Sao Paulo. From these patients, 20 were male $(66.7 \%)$ and 10 were female (33.3\%) with a mean age of 26.6 years (range 6-47 years). The serological screening for the routinely tested viruses transmitted by blood transfusion revealed a seroprevalence of $23.3 \%(\mathrm{n}=7 / 30)$ for anti-HCV $\operatorname{IgG}$ and $16.7 \%$ of anti-Hbs Ag $(n=7 / 30)$.

Between September-October, 2018, blood samples were collected individually from each patient during monthly examinations at the outpatient clinic. All patients included in this study were transfusion-dependent and represented a total of $73.2 \%$ of all thalassemia patients treated in our institution. Approximately $6 \mathrm{~mL}$ of whole blood were collected in a sterile vacutainer tube (Beckton-Dickinson, NJ, USA), after individual informed consent. The samples were initially centrifuged at low speed for plasma separation $(1,931 \times \mathrm{g}, 10 \mathrm{~min})$ and if not immediately processed they were stored at $-80{ }^{\circ} \mathrm{C}$. The study was approved by the Institutional Ethics Committee of the University Hospital at the Faculty of Medicine of Ribeirao Preto, University of Sao Paulo, Brazil (process N HCRP-12196/2018).

\section{Sample preparation and Illumina sequencing}

Initially, $600 \mu \mathrm{L}$ of plasma obtained from each patient were pre-treated with Turbo DNase (Turbo DNA-free kit, Thermo Fisher Scientific, Waltham, MA, USA ) in order to remove free host/bacterial DNA in the analyzed samples (DNAse incubation at $37{ }^{\circ} \mathrm{C}$ for $30 \mathrm{~min}$ ). After DNase inactivation, five individual samples were mixed in a single pool. The total pool volume $(3 \mathrm{~mL})$ was extracted using the High Pure Viral Nucleic Acid Large Volume Kit (Roche, Sao Paulo, Brazil) following the manufacturer's instructions with minor modifications: the polyA carrier RNA from the kit was replaced by the neutral carrier i.e. GenElute Linear Polyacrylamide carrier (LPA) (Merck \& Co., Kenilworth, New Jersey, USA) and Isopropanol was added to the Binding buffer before the filtration step. After column extraction, viral nucleic acids were submitted to reverse transcription using the Superscript III First-Strand Synthesis System (ThermoFisher Scientific,Waltham, MA, USA). Amplification of synthesized cDNA was performed using the QuantiTect Whole Transcriptome Kit (QIAGEN, Hilden, Germany). The preparation of libraries for sequencing was performed using the Nextera DNA Flex Library Preparation Kit (Illumina, San Diego, CA, USA) and the Nextera DNA CD Indexes kit following the manufacturer's instructions. The sequencing the dual-indexed libraries was carried out with an Illumina NextSeq 550 sequencer using the NextSeq 
High Output Kit v.2.5, 300 cycles (Illumina, San Diego, CA, USA) following the manufacturer's instructions. The sensitivity of viral identification sequencing was evaluated using pools containing clinically relevant DNA and RNA viral agents with varying levels of viral load, as described previously ${ }^{16}$.

\section{Bioinformatics processing of the obtained sequencing data}

Sequence reads were initially processed for quality examination using FastQC (v. 0.11.8, Babraham Institute, Cambridge, UK). The low-quality reads, sequences with ambiguous bases and duplicated reads were removed using PRINSEQ (v. 0.20.4, SDSU, San Diego, CA, USA). To remove any host-related sequences and to retain as much viral sequences as possible, we used DeconSeq (v. 0.4.3, SDSU, San Diego, CA, USA). The remaining reads were taxonomically analyzed using Kraken (v. 2, Johns Hopkins University, Baltimore, MD, USA). The unclassified sequences were analyzed using Blastn and Blastx. Assembly was performed by SPAdes (v. 3.13.0, St. Petersburg State University, CAB, St. Petersburg, Russia). All generated contigs were analyzed using Blastn with nucleotide database obtained by the NCBI and an E-value cutoff of $10^{-3}$. For protein similarity evaluation, we used DIAMOND (v.2.0.6, University of Tübingen, Tübingen, Germany). The presence of environmental or other contaminants/possible misclassifications was evaluated using the P-DiP pipeline as previously established ${ }^{17}$.

\section{Phylogenetic analysis of the most important viral contigs}

Phylogenetic analysis of the consensus contigs was performed using datasets of complete or partial genomes obtained from NCBI (National Center for Biotechnology Information). Multiple alignment was performed using MAFFT (v.7.450, Kyoto University, Kyoto, Japan) and the phylogenetic signal was verified using TREE-PUZZLE v. 5.3 (Max-Planck-Institute, Berlin, Germany). The reconstruction of the trees was performed with IQ-TREE (v. 1.6.8, CIBV, Vienna, Austria) applying the Maximum Likelihood method with a statistical support of 10,000 bootstrap replicates. The phylogenetic trees were visualized with FigTree (v. 1.4, IEB, University of Edinburgh, Edinburgh, UK). Only bootstrap values of above $75 \%$ were considered statistically significant and were represented in important phylogenetic branches.

\section{RESULTS}

The Illumina sequencing generated a total of $808,427,182$ reads in plasma samples obtained from patients with beta thalassemia. We determined an average of 134 million reads with nucleotide lengths of $150 \mathrm{bp}$ for each plasma pool (range: $119-160 \times 10^{6} \mathrm{reads} / \mathrm{pool}$ ) (Table 1). After trimming, an average of $13.5 \times 10^{6}$ reads (10.48\%) were eliminated from five pools, while one pool (number 6) showed removal of $82 \times 10^{6}$ million reads ( $50.9 \%$ from its total reads). After taxonomic classification, the classified sequences (range: $73-119 \times 10^{6} \mathrm{reads} / \mathrm{pool}$ ) were kept for de novo assembly and Blastn/Blastx analysis. On average, viral sequences corresponded to $0.023 \%$ of reads per pool (range: 0.002 to $0.11 \%$ ). The unclassified sequences corresponded to a medium of $5.82 \%$ of the total reads (range: $5.19 \%-6.24 \%$ ). From the classified viral sequences, the maximum contig length was 3,786 bp and belonged to HCV (start position $3,441 \mathrm{bp}$ ends $7,158 \mathrm{bp}$ when aligned with the reference HCV genome NC_038882. This region encompassed the entire NS3, NS4A and NS4B genes, and partially to NS5A gene). We observed that $\sim 93 \%$ of the classified contigs were assigned as human sequences. From the known viruses, we detected three major viral families: Anelloviridae, Flaviviridae and Hepadnaviridae. Most of the viral reads corresponded to anelloviruses and were mainly assigned to Torque teno virus (TTV) (> 95\%) from the Alphatorquetenovirus genus, detected in each pool. Other Anelloviridae family genus including Gammatorquevirus (Torque teno midi virus, TTMDV) and Betatorquevirus

Table 1 - Workflow of the sequences analysis, from raw data to assignment.

\begin{tabular}{ccccccc}
\hline Pool number & Total reads & $\begin{array}{c}\text { Reads after } \\
\text { trimming }\end{array}$ & Classified reads & Viral reads & Viral Reads (\%) & $\begin{array}{c}\text { Unclassified } \\
\text { reads }\end{array}$ \\
\hline Pool 1 & $126,241,206$ & $112,680,449$ & $106,827,862$ & 2,642 & $0.002 \%$ & $5,852,587$ \\
Pool 2 & $140,189,184$ & $120,822,545$ & $113,278,558$ & 141,841 & $0.11 \%$ & $7,543,987$ \\
Pool 3 & $123,039,880$ & $109,175,346$ & $102,493,334$ & 26,556 & $0.02 \%$ & $6,682,012$ \\
Pool 4 & $119,051,092$ & $107,960,664$ & $101,267,675$ & 3,414 & $0.003 \%$ & $6,692,989$ \\
Pool 5 & $139,336,406$ & $128,896,726$ & $119,770,867$ & 3,673 & $0.003 \%$ & $7,125,859$ \\
Pool 6 & $160,569,414$ & $78,111,788$ & $73,753,074$ & 3,555 & $0.002 \%$ & $4,358,714$ \\
\hline
\end{tabular}


(Torque teno mini virus, TTMV), were also represented (1.5-16.7\% of all anelloviruses present). Human pegivirus- 1 (HPgV-1, formerly known as GB virus C or Hepatitis G virus) was also found in three out of six plasma pools with a particularly high number of reads in pool number 2 $(158,790$ reads) (Table 2). The performed phylogenetic reconstruction of the largest $\mathrm{HPgV}-1$ contig $(1,360 \mathrm{bp}$, sequence starting at position 2,228 bp and ending at position at 3,554 bp when aligned with HPgV-1 genome NC_001710 corresponding to the p7-NS2 gene), demonstrated that it belongs to genotype 2 (subgenotype 2A) (Figure 1).

We have also detected a large number of sequences $(1,390$ reads) in one of the pools (pool 6, Table 2) corresponding to $\mathrm{HCV}$ (family Flaviviridae). The detection of $\mathrm{HCV}$ nucleic acids was related to the presence of $\mathrm{HCV}$ viremia in one of the samples of the pool (viral load of $\mathrm{HCV}, 56,793 \mathrm{IU} / \mathrm{mL}$ ). The performed phylogenetic analysis of the obtained 3,786 bp contigs demonstrated that it belongs to HCV genotype 1A (Figure 1). The established bioinformatics pipeline was also able to detect 8 reads belonging to HBV (Hepadnaviridae family) in one pool (pool 4). The phylogenetic analysis of the obtained HBV contigs (244 bp, sequence starting at position 1,610 bp and ending at position $1,853 \mathrm{bp}$ aligned with reference genome NC_003977.2 and corresponding partially to the genomic region of the $\mathrm{X}$ protein) demonstrated that it belongs to genotype $\mathrm{A}$. The consensus sequences used in the phylogenetic analyses were deposited under the following

Table 2 - Number of reads of the main viral families detected by the applied metagenomic pipeline in plasma samples from patients with beta-thalassemia.

\begin{tabular}{|c|c|c|c|c|c|c|}
\hline Samples & Pool 1 & Pool 2 & Pool 3 & Pool 4 & Pool 5 & Pool 6 \\
\hline Anelloviridae & 1,794 & 13,126 & 42,954 & 4,480 & 4,081 & 3,088 \\
\hline Alphatorquevirus & 1,724 & 11,384 & 41,587 & 3,912 & 3,516 & 3,012 \\
\hline Gamatorquevirus & 52 & 1,232 & 827 & 408 & 313 & 23 \\
\hline Betatorquevirus & 5 & 251 & 82 & 42 & 132 & 17 \\
\hline HPgV-1 (GBV-C) & - & 158,790 & 5 & 26 & - & - \\
\hline Hepatitis B & - & - & - & 8 & - & - \\
\hline Hepatitis C & - & - & - & - & - & 1,390 \\
\hline
\end{tabular}
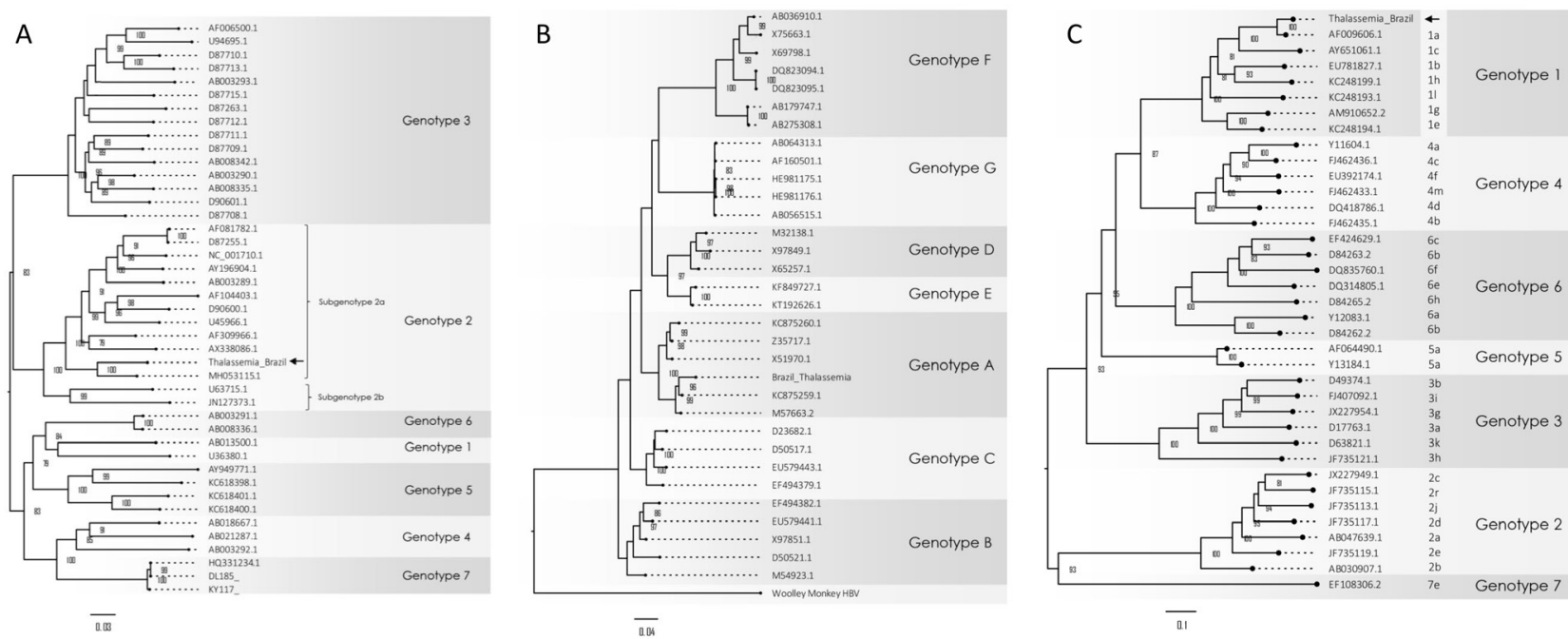

Figure 1 - Phylogenetic trees of the most abundant viruses detected by viral metagenomic analysis in patients with $\beta$-thalassemia major. The phylogenetic trees were reconstructed using the General Time Reversible (GTR) and Transversion Nucleotide Substitution model (TVM) selected by the ModelFinder through Bayesian Information Criteria (BIC), as implemented by IQtree software v. 1.6.2. The Maximum-Likelihood method was used for tree topology reconstruction and for statistical support we used 10,000 ultrafast bootstrap replicates. Only bootstrap values above $75 \%$ were maintained at important tree branches: A) Phylogenetic tree of the contig of Human Pegivirus-1, which was classified as subgenotype 2A of the main genotype 2 that is commonly found in patients with high risk of parenteral exposure. The $\mathrm{GTR}+\mathrm{F}+\mathrm{R} 4$ nucleotide substitution model was used for tree reconstruction; $\mathrm{B}$ ) Taxonomic classification of the HBV contig, which clustered within genotype A that is widely spread in Brazil. For tree reconstruction the $\mathrm{TVM}+\mathrm{F}+\mathrm{I}+\mathrm{G} 4$ nucleotide substitution model was used; C) Phylogenetic tree of HCV. The arrow shows the position of the assembled contig within the subgenotype $1 \mathrm{~A}$ of the main HCV genotype 1 . For the tree reconstruction, the GTR+F+R5 nucleotide substitution model was used. 
numbers in the NCBI i.e., HCV (MT596912), HPgV-1 (MT596914) and HBV (MT596913).

\section{DISCUSSION}

In this study, performed among chronically transfused Brazilian patients with beta-thalassemia, we evaluated their virome by mHTS. By applying this approach, we tried to identify emerging or unsuspected viral agents which can threaten the transfusion safety. The main reasons to perform this study are the high-risk of the thalassemic patients to acquire parenteral infections and the endemicity of the studied region for arboviruses and other tropical infections which can be transmitted via blood transfusion ${ }^{18,19}$.

In all of the screened pools we found viruses showing persistent viremia, thus demonstrating that transmission can occur parenterally. Among them, the most abundant were the members of the Anelloviridae family, represented by the three major genera: Alpha-, Beta- and Gammatorquetenvirus. By far, this is the most abundant group of viruses found in blood samples analyzed by viral metagenomics ${ }^{20-22}$. The obtained results are in agreement with the fact that anelloviruses are characterized by a high level of genome diversity and high prevalences in the human population which in some cases can reach $100 \%{ }^{23,24}$. Despite the high anellovirus prevalence, the way these viruses interact with the hosts is not elucidated.

Another widely distributed virus, which was found by the metagenome pipeline was the $\mathrm{HPgV}-1$, showing an elevated number of reads in one of the tested pools. Further phylogenetic analysis of the major contigs confirmed the presence of $\mathrm{HPgV}-1$ genotype 2 (subgenotype $2 \mathrm{~A}$ ), which is the most prevalent genotype in Brazil ${ }^{25,26}$ that is efficiently transmitted by blood transfusion. Similar to the anelloviruses, $\mathrm{HPgV}-1$ is highly prevalent in different patient groups, especially in patients with high parenteral exposure ${ }^{27-30}$. This is in accordance with our findings of a high number of $\mathrm{HPgV}$ 1 contigs detected among patients with beta-thalassemia. Our results are similar to data from the literature which demonstrated that HPgV-1 and anelloviruses are the most commonly found commensal viruses detected in human plasma $^{31}$. In this respect, these commensal viruses were also most frequently identified by viral metagenomic approaches in blood donations obtained from healthy donors ${ }^{31,32}$. In general, healthy blood donors are a suitable control group that can also be used to compare our results in order to evaluate the impact of these commensal viruses on blood transfusion. Although we did not implement such a control, we believe that the composition of the commensal virome between patients and donors might be quite similar based on similarities obtained from the literature data ${ }^{31,32}$.
On the other hand, important parenterally-transmitted viruses like HCV and HBV were also identified which differs from the normal blood donor virome. The detection of $\mathrm{HCV}$ reads in one of the tested pools was due to the high seroprevalence of anti-HCV IgG in the tested group of patients $(23.3 \%)$. The phylogenetic analysis of the largest HCV contig (3,786 bp) demonstrated that it belongs to genotype 1A. This finding is in accordance with the literature, pointing out that genotype $1 \mathrm{~A}$ is the most prevalent $\mathrm{HCV}$ genotype in $\mathrm{Brazi}^{33}$. We also detected a few reads belonging to HBV. The HBV contig was composed of $244 \mathrm{bp}$ and although relatively small, it was further classified as belonging to HBV genotype A, which is also a common Brazilian genotype ${ }^{34}$. The identification of few HBV reads probably reflects low viral loads and that the sensitivity of the applied sequencing is adequate for the identification of low viral load infections. This was supported on one hand by our previous studies which sensitively identify underrepresented viruses in artificially spiked pools ${ }^{16}$, and on the other by the extraordinary deepness of the applied sequencing (around 200 million reads for each tested pool) which possibly enabled the detection of infections with low viral loads. In addition, the obtained results show that the presence of HCV-RNA and HBV-DNA in multiple - transfused patients with beta-thalassemia major is in accordance with the genotype distribution of these two viruses in Brazil, and that the used HTS pipeline was capable of determining the presence of active viremia of chronic viral infections.

A shortcoming was that annotated viral sequences were not confirmed by direct molecular methods like PCR. However, metagenomics has a high diagnostic potential due to the correlation between the percentage of viral reads of a given virus and its viral load in the examined sample ${ }^{35,36}$. Nevertheless, sequence reads of only highly represented agents were taken into account, except for HBV, which was previously confirmed by serological analysis. According to some authors, metagenomics could be considered even superior to direct molecular methods when working with highly diverse viral taxa such as the Anellovirus family for which exact primer matching and efficient amplification are difficult to be achieved ${ }^{36-38}$.

In this study, we did not characterize the sequence of any new virus that can be regarded as an emerging threat to multiple-transfused patients with beta-thalassemia. In general, the identification of emerging viruses is dependent on nucleotide alignments with known viruses and it is difficult to taxonomically classify phylogenetically distant viral agents ${ }^{20}$. However, we identified some sequences with low nucleotide sequence coverage (20-30\%) belonging to the Marseilleviridae, Phycodnaviridae and Mimiviridae 
families which represent giant viruses some of which have been putatively transmitted by blood transfusion ${ }^{39}$. Due to the low percentage of genomic coverage, we could not attribute with certainty that these viral agents are emerging viruses threatening the transfusion policies in Brazil. Therefore, we believe that there is a need for improvement of the current bioinformatics approach to be able to classify and identify sequences of phylogenetically distant viruses ${ }^{40}$.

\section{CONCLUSION}

In conclusion, this study examined the viral landscape in patients with beta-thalassemia. We detected only known commensal and parenterally-transmitted viruses belonging to different viral families in high-risk patients with beta-thalassemia major. Our study demonstrates that viral metagenomic approaches represent an interesting tool for the evaluation of viral diversity in patients subjected to multiple transfusions, reinforcing its relevance for the discovery of viral agents that can potentially threaten the transfusion safety.

\section{ACKNOWLEDGMENTS}

The authors would like to acknowledge all patients who donated their samples for this study. We also would like to thank Carolina Oliveira de Almeida Pereira and Salua Ingrid Jorge Miranda Ferreira for their help in sample collection. The authors also thank Sandra Navarro Bresciani for the artwork and Antoaneta Ivanova Slavova (in memoriam) for eternal advice. The research was supported by the Sao Paulo Research Foundation (FAPESP: 17/23205-8, 2018/15826-5, 19/07861-8, 19/08528-0) and Conselho Nacional do Desenvolvimento Cientifico e Tecnologico, Brazil (INCTC: 465539/2014-9, 455503/2014-1).

\section{CONFLICT OF INTERESTS}

The authors have no conflict of interests to declare.

\section{REFERENCES}

1. Ainley LI, Hewitt PE. Haematology patients and the risk of transfusion transmitted infection. Br J Haematol. 2018;180:473-83.

2. Corrêa AS, Lamarão LM, Vieira PC, Castro RB, Almeida NC, Castro JA, et al. Prevalence, incidence and residual risk of transfusion-transmitted HBV infection before and after the implementation of HBV-NAT in northern Brazil. PLoS One. 2018;13:e0208414.
3. Candotti D, Assennato SM, Laperche S, Allain JP, LevicnikStezinar S. Multiple HBV transfusion transmissions from undetected occult infections: revising the minimal infectious dose. Gut. 2019;68:313-21.

4. Dunford L, Carr MJ, Dean J, Waters A, Nguyen LT, Ta Thi TH, et al. Hepatitis $\mathrm{C}$ virus in Vietnam: high prevalence of infection in dialysis and multi-transfused patients involving diverse and novel virus variants. PLoS One. 2012;7:e41266.

5. Cappy P, Barlet V, Lucas Q, Tinard X, Pillonel J, Gross S, et al. Transfusion of HIV-infected blood products despite highly sensitive nucleic acid testing. Transfusion. 2019;59:2046-53.

6. Stramer SL, Hollinger FB, Katz LM, Kleinman S, Metzel PS, Gregory KR, et al. Emerging infectious disease agents and their potential threat to transfusion safety. Transfusion. 2009;49 Suppl 2:1S-29S.

7. Sabino EC, Loureiro P, Lopes ME, Capuani L, McClure C, Chowdhury D, et al. Transfusion-transmitted dengue and associated clinical symptoms during the 2012 epidemic in Brazil. J Infect Dis. 2016;213:694-702.

8. Karim F, Nasir N, Moiz B. Transfusion transmitted dengue: one donor infects two patients. Transfus Apher Sci. 2017;56:151-3.

9. Motta IJ, Spencer BR, Silva SG, Arruda MB, Dobbin JA, Gonzaga YB, et al. Evidence for transmission of Zika virus by platelet transfusion. N Engl J Med. 2016;375:1101-3.

10. Riveiro-Barciela M, Sauleda S, Quer J, Salvador F, Gregori J, Pirón M, et al. Red blood cell transfusion-transmitted acute hepatitis $\mathrm{E}$ in an immunocompetent subject in Europe: a case report. Transfusion. 2017;57:244-7.

11. Ticehurst JR, Pisanic N, Forman MS, Ordak C, Heaney CD, Ong E, et al. Probable transmission of hepatitis E virus (HEV) via transfusion in the United States. Transfusion. 2019;59:1024-34.

12. Ma YY, Guo Y, Zhao X, Wang Z, Lv MM, Yan QP, et al. Human parvovirus PARV4 in plasma pools of Chinese origin. Vox Sang. 2012;103:183-5.

13. Makroo RN, Arora JS, Chowdhry M, Bhatia A, Thakur UK, Minimol A. Red cell alloimmunization and infectious marker status (human immunodeficiency virus, hepatitis B virus and hepatitis $\mathrm{C}$ virus) in multiply transfused thalassemia patients of North India. Indian J Pathol Microbiol. 2013;56:378-83.

14. Sauvage V, Ar Gouilh M, Cheval J, Muth E, Pariente K, Burguiere A, et al. A member of a new Picornaviridae genus is shed in pig feces. J Virol. 2012;86:10036-46.

15. Zhang YZ, Shi M, Holmes EC. Using metagenomics to characterize an expanding virosphere. Cell. 2018;172:1168-72.

16. Valença IN, Silva-Pinto AC, Silva Júnior WA, Covas DT, Kashima S, Slavov SN. Viral metagenomics in Brazilian multiply transfused patients with sickle cell disease as an indicator for blood transfusion safety. Transfus Clin Biol. 2020;27:237-42.

17. Zapatka M, Borozan I, Brewer DS, Iskar M, Grundhoff A, Alawi $\mathrm{M}$, et al. The landscape of viral associations in human cancers. Nat Genet. 2020;52:320-30. 
18. Slavov SN, Hespanhol MR, Rodrigues ES, Levi JE, Ubiali E, Covas DT, et al. Zika virus RNA detection in asymptomatic blood donors during an outbreak in the northeast region of São Paulo State, Brazil, 2016. Transfusion. 2017;57:2897-901.

19. Pereira BB, Darrigo Junior LG, Costa TC, Felix AC, Simoes $\mathrm{BP}$, Stracieri $\mathrm{AB}$, et al. Prolonged viremia in dengue virus infection in hematopoietic stem cell transplant recipients and patients with hematological malignancies. Transpl Infect Dis. 2017;19:e12721.

20. Sauvage V, Laperche S, Cheval J, Muth E, Dubois M, Boizeau L, et al. Viral metagenomics applied to blood donors and recipients at high risk for blood-borne infections. Blood Transfus. 2016;14:400-7.

21. Bal A., Sarkozy C, Josset L, Cheynet V, Oriol G, Becker J, et al. Metagenomic next-generation sequencing reveals individual composition and dynamics of Anelloviruses during autologous stem cell transplant recipient management. Viruses. 2018;10:633.

22. Pan S, Yu T, Wang Y, Lu R, Wang H, Xie Y, et al. Identification of a Torque Teno Mini Virus (TTMV) in Hodgkin's lymphoma patients. Front Microbiol. 2018;9:1680.

23. Biagini P, Gallian P, Cantaloube JF, Attoui H, de Micco P, Lambalerrie X. Distribution and genetic analysis of TTV and TTMV major phylogenetic groups in French blood donors. J Med Virol. 2006;78:298-304.

24. Al-Qahtani AA, Alabsi ES, AbuOdeh R, Thalib L, El Zowalaty ME, Nasrallah GK. Prevalence of anelloviruses (TTV, TTMDV, and TTMV) in healthy blood donors and in patients infected with HBV or HCV in Qatar. Virol J. 2016;13:208.

25. Ramos Filho R, Carneiro MA, Teles AS, Dias MA, Cardoso $\mathrm{DD}$, Lampe $\mathrm{E}$, et al. $\mathrm{GB}$ virus $\mathrm{C} /$ hepatitis $\mathrm{G}$ virus infection in dialysis patients and kidney transplant recipients in Central Brazil. Mem Inst Oswaldo Cruz. 2004;99:639-43.

26. Alcalde R, Nishiya A, Casseb J, Inocêncio L, Fonseca LA, Duarte AJ. Prevalence and distribution of the GBV-C/HGV among HIV-1-infected patients under anti-retroviral therapy. Virus Res. 2010;151:148-52.

27. Seifried C, Weber M, Bialleck H, Seifried E, Schrezenmeier $\mathrm{H}$, Roth KW, et al. High prevalence of GBV-C/HGV among relatives of GBV-C/HGV-positive blood donors in blood recipients and in patients with aplastic anemia. Transfusion. 2004;44:268-74.

28. Aster V, König J, Stanková M, Rozsypal H, Procházka B. Prevalence of GBV-C/HGV (HGV) in HIV-infected patients and potential influence of co-infection on the course of the disease. Klin Mikrobiol Infekc Lek. 2005;11:199-1203.

29. Motta LD, Nishiya AS, Finger-Jardim F, Barral MF, Silva CM, Nader MM, et al. Prevalence of human pegivirus $(\mathrm{HPgV})$ infection in patients carrying HIV-1C or non-C in southern Brazil. J Med Virol. 2016;88:2106-14

30. Santos LM, Lobato RC, Barral MF, Gonçalves CV, Hora VP,
Martinez AM. Prevalence and vertical transmission of human pegivirus among pregnant women infected with HIV. Int J Gynaecol Obstet. 2017;138:113-8.

31. Zhang W, Li L, Deng X, Blümel J, Nübling CM, Hunfeld A, et al. Viral nucleic acids in human plasma pools. Transfusion. 2016;56:2248-55.

32. Li G, Zhou Z, Yao L, Xu Y, Wang L, Fan X. Full annotation of serum virome in Chinese blood donors with elevated alanine aminotransferase levels. Transfusion. 2019;59:3177-85.

33. Bruggmann P, Berg T, Øvrehus AL, Moreno C, Brandão Mello $\mathrm{CE}$, Roudout-Thoroval $\mathrm{F}$, et al. Historical epidemiology of hepatitis $\mathrm{C}$ virus $(\mathrm{HCV})$ in selected countries. J Viral Hepat. 2014;21 Suppl 1:5-33.

34. Lampe E, Mello FC, Espírito-Santo MP, Oliveira CM, Bertolini DA, Gonçales NS, et al. Nationwide overview of the distribution of hepatitis B virus genotypes in Brazil: a 1000-sample multicentre study. J Gen Virol. 2017;98:1389-98.

35. Graf EH, Simmon KE, Tardif KD, Hymas W, Flygare S, Eilbeck K, et al. Unbiased detection of respiratory viruses by use of RNA sequencing-based metagenomics: a systematic comparison to a commercial PCR panel. J Clin Microbiol. 2016;54:1000-7.

36. Greninger AL, Zerr DM, Qin X, Adler AL, Sampoleo R, Kuypers JM, et al. Rapid metagenomic next-generation sequencing during an investigation of hospital-acquired human parainfluenza virus 3 infections. J Clin Microbiol. 2016;55:177-82.

37. Sedlak RH, Nguyen T, Palileo I, Jerome KR, Kuypers J. Superiority of Digital Reverse Transcription-PCR (RT-PCR) over real-time RT-PCR for quantitation of highly divergent human rhinoviruses. J Clin Microbiol. 2017;55:442-9.

38. Greninger AL. The challenge of diagnostic metagenomics. Expert Rev Mol Diagn. 2018;18:605-15.

39. Popgeorgiev N, Colson P, Thuret I, Chiarioni J, Gallian P, Raoult $\mathrm{D}$, et al. Marseillevirus prevalence in multitransfused patients suggests blood transmission. J Clin Virol. 2013;58:722-5.

40. Zhang YZ, Shi M, Holmes EC. Using metagenomics to characterize an expanding virosphere. Cell. 2018;172:1168-72.

\section{ERRATUM}

Page 1, where it reads:

Rafael Bezerra dos Santos

Should be read:

Rafael dos Santos Bezerra

http://dx.doi.org/10.1590/s1678-9946202163040err 